\section{Smoking as a confounder of the association of suicidality with serum lipid levels}

A meta-analysis by $\mathrm{Wu}$ and colleagues ${ }^{1}$ reported an inverse association between serum lipid levels and suicidality. The authors compared total serum cholesterol (TC), high(HDL-C) and low-density lipoprotein cholesterol (LDL-C) and triglyceride (TG) concentrations between individuals with psychiatric disorders (depression, schizophrenia, personality disorders, drug and alcohol addiction) with or without suicidality (ideation, threatened suicide, suicide attempts, suicide completion) to healthy controls. They found that suicidal persons had significantly lower serum TC, LDL-C and TG than nonsuicidal persons. There was no overall difference between suicidal patients and healthy controls for HDL-C with the exception of individuals with schizophrenia compared with controls.

The authors mentioned that some included studies did not adjust for important confounders, such as alcohol consumption or smoking. Smoking is the most prevalent health disorder worldwide, it is an independent risk factor for all aspects of suicide behaviour (ideation, wish to die, attempt) ${ }^{2}$ and it is dose-dependently associated with suicide. ${ }^{3}$ Smoking increases the risk of suicide attempt when controlling for all other potential confounders, among which prior suicide attempt is the strongest predictor of a subsequent suicide attempt., ${ }^{4,5}$ Moreover, smoking is strongly comorbid with psychiatric disorders, ${ }^{6}$ particularly schizoaffective disorders, and it can be hypothesized that smoking is an additive risk factor for suiciderelated behaviours among the psychiatrically ill.

At the level of serum lipids, the most characteristic association with smoking is the reduced HDL-C $C^{7,8}$ even in healthy youth; ${ }^{9}$ HDL-C becomes normal $^{7}$ or increases ${ }^{10}$ when smokers quit. Thus, adjusting for smoking status could potentially cancel the association between HDL-C and suicidality observed among suicidal patients with schizophrenia, suicidal patients with other disorders and controls. Similarly, because smoking is frequently associated with elevated TG and metabolic syndrome, ${ }^{8}$ controlling for smoking would reduce the difference between suicidal and nonsuicidal patients or controls. It is therefore of major interest to control for smoking behaviour when the association of suicidality with serum lipids is assessed.

Ivan Berlin, MD, PhD; Amandine Luquiens, MD, PhD; Henri-Jean Aubin, MD, PhD

Affiliations: From the Département de pharmacologie, Hôpital Pitié-Salpêtrière Université P. \& M. Curie, Paris, France; and the Département de psychiatrie et d'addictologie, Villejuif, France.

\section{DOI: 10.1503/jpn.150361}

\section{References}

1. Wu S, Ding Y, Wu F, et al. Serum lipid levels and suicidality: a meta-analysis of 65 epidemiological studies. J Psychiatry Neurosci 2016;41:56-69.

2. Covey LS, Berlin I, Hu MC, et al. Smoking and suicidal behaviours in a sample of US adults with low mood: a retrospective analysis of longitudinal data. BMJ Open 2012;2:e000876.

3. Li D, Yang X, Ge Z, et al. Cigarette smoking and risk of completed suicide: a meta-analysis of prospective cohort studies. J Psychiatr Res 2012;46:1257-66.

4. Riala K, Taanila A, Hakko H, et al. Longitudinal smoking habits as risk factors for early-onset and repetitive suicide attempts: the Northern Finland 1966 Birth Cohort Study. Ann Epidemiol 2009;19: 329-35.

5. Berlin I, Hakes KH, Hu MC, et al. Tobacco use and suicide attempt: longitudinal analysis with retrospective reports. PLOS ONE 2015;10:e122607.

6. Lasser K, Boyd W, Woolhandler S, et al. Smoking and mental illness. A population-based prevalence study. JAMA 2000;284:2606-10.

7. Bakhru A, Erlinger TP. Smoking cessation and cardiovascular disease risk factors: results from the Third National Health and Nutrition Examination Survey. PLoS Med 2005;2:e160.
8. Berlin I, Lin S, Lima JA, et al. Smoking status and metabolic syndrome in the multi-ethnic study of atherosclerosis. A cross-sectional study. Tob Induc Dis 2012;10:9.

9. Craig WY, Palomaki GE, Johnson M, et al. Cigarette smoking-associated changes in blood lipid and lipoprotein levels in the 8- to19-year-old age group: a meta-analysis. Pediatrics 1990;85:155-8.

10. Forey BA, Frys JS, Lee PN, et al. The effect of quitting smoking on HDLcholesterol - a review based on withinsubject changes. Biomarker Research 2013; 1:26.

\section{Author response}

We thank Berlin and colleagues for their comments on our study. ${ }^{1}$ The letter focused on smoking as a confounder of the association between suicidality and serum lipid levels. They commented that controlling for smoking would reduce the difference of serum lipid levels between suicidal and nonsuicidal patients or controls. This topic is very important, and we welcome this discussion.

We agree that smoking, as a demographic characteristic, plays an important role in suicidality. However, for this to have been accounted for in the findings, smoking must have been related to serum lipid levels and must have been associated with risk of suicidality. Regarding the former, nobody would disagree with the statement that smoking is associated with serum lipid levels; however, with respect to the latter, to be considered a confounder the association of smoking with suicidality must be independent from its association with serum lipid level. We agree that smoking may be related to suicidality, but as far as we have been able to find out, there is no evidence that smoking per se is an independent causal risk factor that is not linked to the change of serum lipid levels.

In addition, the associations among smoking, mental illness and suicide remain unknown. Some studies have found that smoking was not significantly associated with suicidal 
behaviour after adjusting for mental disorders. ${ }^{2,3}$ Based on these findings, the inference that smoking may be a confounder of the association between suicidality and serum lipid levels lacks sufficient evidence.

Our meta-analysis was based on a wide range of published articles (65 epidemiological studies), and the inverse associations between serum lipid levels and suicidality appeared to be consistent across most studies. Thus, the results of our study are sound and reliable.

Shunquan Wu, MD; Jun Hou, PhD;

Panyong Mao, PhD

Affiliations: From the Research Center for Clinical and Translational Medicine, the 302nd Hospital of PLA, Beijing, China.

DOI: 10.1503/jpn.150366

\section{References}

1. Wu S, Ding Y, Wu F, et al. Serum lipid levels and suicidality: a meta-analysis of 65 epidemiological studies. J Psychiatry Neurosci 2016;41:56-69.

2. Kessler RC, Berglund PA, Borges G, et al. Smoking and suicidal behaviors in the National Comorbidity Survey: replication. J Nerv Ment Dis 2007;195:369-77.

3. McGee R, Williams S, Nada-Raja S. Is cigarette smoking associated with suicidal ideation among young people? Am J Psychiatry 2005;162:619-20. 